\title{
Prevalence of Crytpococcal Infection in Patients Clinically Diagnosed to Have Meningitis in Ethiopia
}

\author{
Adane Bitew $^{1}$, *, Mulu Hassen ${ }^{1}$, Tigist Getachew ${ }^{1}$, Surafel Fentaw ${ }^{2}$ \\ ${ }^{1}$ Department of Medical Laboratory Sciences, College of Health Sciences, Addis Ababa University, Addis Ababa, Ethiopia \\ ${ }^{2}$ Department of Clinical Bacteriology and Mycology, Ethiopian Public Health Institute, Addis Ababa, Ethiopia \\ Email address: \\ bitewadane@gmail.com (A. Bitew), muluh2233@gmail.com (M. Hassen), tigistgetachew627@gmail.com (T. Getachew), \\ sura4f@gmail.com (S. Fentaw) \\ ${ }^{*}$ Corresponding author
}

\section{To cite this article:}

Adane Bitew, Mulu Hassen, Tigist Getachew, Surafel Fentaw. Prevalence of Crytpococcal Infection in Patients Clinically Diagnosed to Have Meningitis in Ethiopia. Clinical Medicine Research. Vol. 5, No. 4, 2016, pp. 73-76. doi: 10.11648/j.cmr.20160504.12

Received: May 21, 2016; Accepted: May 31, 2016; Published: June 14, 2016

\begin{abstract}
Cryptococcal meningitis is a fungal infection that causes significant morbidity and mortality worldwide. However, its incidence in Ethiopia is poorly known. The present study was undertaken to determine cryptococcal meningitis in Ethiopian patients diagnosed to have meningitis. Cerebrospinal fluid was collected from 201 patients with meningitis attending two tertiary hospitals for the isolation and characterization of major bacterial pathogens implicated in causing meningitis. Left over sample of each patient was screened for cryptococal antigen using lateral flow Assay. Of the total number of 201 patients 17 (8.5\%) were positive for cryptococcal antigenemia of which $8(47.1 \%)$ were male patients while $9(52.9 \%)$ were female patients. In conclusion a high prevalence of cryptococcal meningitis in the present study urges detection and /or isolation and characterization of the pathogen in patients diagnosed to have meningitis regardless of the immune status. Furthermore, in view of the increasing number of cryptococcal infections, rapid, sensitive, and specific diagnosis of cryptococcal disease has become more important than ever in Ethiopian health institutions.
\end{abstract}

Keywords: Cryptococcus, Meningitis, Ethiopia

\section{Introduction}

Cryptococcal meningitis is an infectious disease that affects predominantly immune compromised hosts causing significant morbidity and mortality. Although cryptococcosis has been recognized for more than 100 years, worldwide spread of HIV infection and other forms of immunesuppression are incriminated as the most important factors for its increase since the 1980's [1]. Currently, it has been estimated that there are about one million new cases of cryptococcosis and at least 600,000 deaths annually worldwide due to HIV-associated cryptococcosis [2]. In subSaharan Africa with the highest HIV prevalence, Cryptococcus species has become the leading cause of meningitis, preceding pneumococcal and meningococcal meningitis [3-9]. The infection accounts $13-44 \%$ of all deaths in HIV infected individuals in this geographical region [10-12].
Although cryptococcal meningitis is generally thought to be associated with immune-compromised patients (i.e., HIVpositive patients and non-HIV-infected immunocompromised patients), recent studies [13-15] showed that its incidence has increased among immunecompetent individuals. Ethiopia being located in sub-Saharan Africa, the morbidly and mortality of cryptococcal meningitis is poorly known. To the best of our knowledge, two case reports $[16,17]$ and three full flagged research articles in HIV infected patients [18-20] have been published with regards to cryptococcal infection.

An accurate diagnosis of cryptococcosis is important for its successful treatment. The cost and long duration of the therapy, the risk of developing adverse drug reactions, and possible interactions with concomitant medications all underline the importance of accurate diagnosis of the condition before commencing therapy. Therefore, early identification of cryptococcal infection regardless of the immune status of a host and clinical manifestations is 
indispensable for appropriate management of the complication.

Demonstrating capsulated yeast by India ink preparation, culture and detection of capsular polysaccharide antigen serologically from various clinical samples (CSF, serum, urine) has been the mainstay for the diagnosis of cryptococcal infections. Although culture is considered as a gold standard for the isolation and characterization of the yeast, fungal culture is not performed in routine clinical laboratory practice in Ethiopia. Currently, the diagnostic tests most commonly used in the developed world for identifying cryptococcal antigen are the latex agglutination test (LA) and the enzyme immunoassay (EIA). These tests are not practiced in clinical laboratories in Ethiopia since they are resource intensive. Therefore, detection of the yeast by less sensitive and non-specific India ink preparation is the commonest test practice in clinical laboratories in Ethiopia. Lack of sensitive and specific test procedures in Ethiopian health institution have been identified as major problem in the under reporting of the infection. Difficulty in differentiating signs and symptoms of cryptococcal meningitis clinically from those of many other forms of meningitis has also hampered the study of cryptococcal meningitis. In line with, the aim of this study was to determine cryptococcal infection in patients diagnosed to have meningitis regardless of their clinical manifestations and immune status.

\section{Materials and Methods}

\subsection{Study Patients}

Patients diagnosed to have meningitis were enrolled between September 2006 and August 2007 from the outpatient department of Tikur Anbessa (Black Lion) and Yekatit twelve teaching hospitals for the isolation and characterization of major bacterial pathogens implicated in causing meningitis. CSF was collected from 201 patients and left over sample was used for the present study.

\subsection{Laboratory Diagnosis}

Left over CSF sample was transferred to the Department of Medical Laboratory sciences and kept in a deep freezer at minus $80^{\circ} \mathrm{C}$ until use. Each sample was then screened for $\mathrm{CrAg}$ using $\mathrm{CrAg}$ Lateral flow assay (Immuno-Mycologics Inc., Oklahoma, USA) as per the instruction of the manufacturer. The Lateral flow assay uses immunochromographic test strips that have been impregnated with monoclonal antibodies against capsular polysaccharide antigens common to fungi in the Cryptococcal species complex (C. neoformans and $C$. gattii). To perform the assay, 40 microliters of CSF was mixed with one drop of LF specimen Diluent (GLF025). Then the white end of a Cryptococcal Antigen Lateral Flow Test Strip (LFCR50) was submerged into the preparation and the result was read after 10 minutes at room temperature. The presence of two lines (test and control) on the test strip regardless of the intensity of the test line were interpreted as positive result, while the presence of a single control line was read as a negative result.

\subsection{Ethical Clearance}

All ethical considerations and obligations were duly addressed and the study was conducted after the approval of the Department Research and Ethical Review Committee (DRERC) of the Department of Medical Laboratory Sciences, College of Health Sciences, Addis Ababa University and the Addis Ababa City Health Bureau. Informed written consent was obtained from participants before data collection. The respondent was given the right to refuse to take part in the study and to withdraw at any time during the study period. Assent form was completed and signed by family member and/or adult guardian for participants under the age of 18 years.

\section{Result and Discussion}

With a declining incidence of AIDS-related cryptococcosis in the Highly Active Antiretroviral Therapy (HAART) era, and increasing use of immunesuppressive agents worldwide [21, 22], non-HIV infected individuals (with or without predisposing factors) have become more frequently infected by the yeast. Ethiopia being a developing nation located in sub-Saharan Africa where anti-retroviral therapy is less available appears to fall into the category of regions with high prevalence of cryptococcosis. Furthermore, in contrast to immunecompromised patients, few reports have described the variety of $C$. neoformans, clinical presentation, cerebrospinal fluid (CSF) parameters and prognosis associated with cryptococcal infections among immunocompetent individuals [13]. To this effect, screening of crytpococcal infection in Ethiopian patients clinically diagnosed to have meningitis regardless of the immune status appears to be of a highest priority. Consequently, in the present study a total of 201 samples were collected from patients clinically diagnosed to have meningitis. Among study subjects, 106 (52.7\%) were males and $96(47.8 \%)$ were females. The ages of study subjects ranged from 1 day to 75 years with a mean age of 26 years. Of the total number of 201 patients $17(8.5 \%)$ were positive for cryptococcal antigenemia of which 8 (4\%) were male patients while $9(4.5 \%)$ were female patients. As shown in table 1, the finding of the present study was comparable with the findings of previous studies conducted in Ethiopia with HIV infected patients [18-20] but much lower than similar studies conducted in AIDS patients in other sub-Saharan African countries [3, 9, 23-28]. Index of suspicion, patient selection and intensity of evaluation, variation in test procedures and type of clinical sample used for detection and/or isolation and characterization the etiologic agent may have attributed to such a variation. 
Table 1. Prevalence of cryptococcal meningitis in HIV patients: review of available literature in sub Saharan African countries.

\begin{tabular}{|c|c|c|c|c|c|c|}
\hline Country & References & $\begin{array}{l}\text { Total cases of } \\
\text { meningitis }\end{array}$ & $\begin{array}{l}\text { Cryptococcal } \\
\text { meningitis, } n(\%)\end{array}$ & Study population & Sample & $\begin{array}{l}\text { method of } \\
\text { identification }\end{array}$ \\
\hline Ethiopia & Seboxa et al [18] & 375 & $31(8.3 \%)$ & HIV patients & $\mathrm{CSF}$ & India ink \& culture \\
\hline Ethiopia & Alemu et al [19] & 369 & $31(8.8 \%)$ & HIV patients & Blood & Serology \\
\hline Ethiopia & Tafese et al [20] & 254 & $26(10.2 \%)$ & HIV patents & Blood & Serology \\
\hline Central African Republic & Bekondi, et al [3] & 215 & $84(39.1)$ & HIV patients & CSF, & culture \& India ink \\
\hline South Africa & Schutte [9] & 44 & $22(50.0)$ & HIV patients & CSF & $\begin{array}{l}\text { India ink, culture \& } \\
\text { serology }\end{array}$ \\
\hline South Africa & Bergemann \& Karstaedt [23] & 106 & $37(34.9)$ & HIV patients & $\mathrm{CSF}$ & India ink \& serology \\
\hline Zimbabwe & Hakim et al, [24] & 170 & $80(47.1)$ & HIV patients & CSF & serology \& India ink \\
\hline South Africa & Silber et al [25] & 41 & $8(19.5)$ & & $\mathrm{CSF}$ & Culture \\
\hline South Africa & Jarvis et al [26] & 492 & $337(68.5)$ & HIV patients & CSF & $\begin{array}{l}\text { culture, India ink \& } \\
\text { serology }\end{array}$ \\
\hline Malawi & Cohen et al [27] & 235 & $110(46.8)$ & HIV patients & $\mathrm{CSF}$ & $\begin{array}{l}\text { culture India ink \& } \\
\text { serology }\end{array}$ \\
\hline Zambia & Mwaba et at [28] & 230 & $210(91 \%)$ & HIV patients & $\mathrm{CSF}$ & India ink\& culture \\
\hline
\end{tabular}

Quite a good number of studies on the prevalence of cryptococcal meningitis on immunocompetent and apparently immunocompitent patients have been conducted. Prevalence of cryptococcal meningitis among immunocompetent and immunocompromised individuals has been conducted by Mahale, et al [29]. A total of 242 CSF samples collected from patients clinically suspected for cryptococcal meningitis were screened for Cryptococcus neoformans irrespective of their immune status. The result of the study depicted an overall prevalence of $8.3 \%$ cryptococcal meningitis and prevalence $16.6 \%$ cryptococcal meningitis among immunocompromised patients. The result of this study was in good agreement with our finding. Similarly, the prevalence of cryptococcis in apparently immunocompetent patients was conducted by Lui et al [13]. Out of 46 patients with Cryptococcosis, twenty patients (43.5\%) were apparently immunocomptetent. The finding of Lui et al [13] is supported by the report of Pappas et al [30] that showed cryptococcosis in non-HIV patients of which $10-40 \%$ were immunocompetent. To determine the prevalence of cryptococcal infection, a retrospective analysis of 326 clinically diagnosed cases with meningitis over a period of five-and-a-half years was carried out by Prasad et al [31] of which fifty-four $(16.6 \%)$ patients were found to have cryptococcal meningitis. Our finding was not in accordance with the findings of Prasad et al [31], which has demonstrated a higher prevalence of cryptococcal meningitis.

As a result of differences in exposure to the pathogen, cryptococcal meningitis is more frequently reported in men than women and rarely from children [4]. The finding of the present study in this regard was not in line with the findings of this study where the number of male and female patients with cryptococcal meningitis was more or less the same (8: 9).

\section{Conclusion}

Since clinical manifestations of cryptococcal meningitis are indistinguishable from other causes of central nervous system infection, all patients clinical diagnosed as having meningitis must be investigated for cryptococcal meningitis irrespective of their immune status. Furthermore, in view of the increasing number of infections, rapid, sensitive, and specific diagnosis of cryptococcal disease has become more important than ever in Ethiopian health institutions.

\section{Limitation of the Study}

Unable to perform other laboratory procedures as the result of inadequate clinical sample was the limitation of this work.

\section{Conflict of Interest}

The authors declare that there is no conflict of interest regarding the publication of this paper.

\section{Acknowledgement}

The authors would like acknowledge all patients that participated in the present study

\section{References}

[1] Dromer F, Mathoulin S, Dupont B, and Laporte A. "Epidemiology of cryptococcosis in France: a 9-year survey (1985- 1993). French Cryptococcosis Study Group." Clin. Infec. Dis 1996, 23: 82-90.

[2] Park BJ, Wannemuehler KA, Marston BJ, Govender N, Pappas PG, et al. Estimation of the current global burden of cryptococcal meningitis among persons living with HIV/AIDS. AIDS 2009; 23: 525-530.

[3] Bekondi C, Bernede C, Passone N, Minssart P, Kamalo C, Mbolidi D, Germani Y. Primary and opportunistic pathogens associated with meningitis in adults in Bangui, Central African Republic, in relation to human immunodeficiency virus serostatus. Int J Infect Dis 2006; 10: 387-395.

[4] Bogaerts J, Rouvroy D, Taelman H, Kagame A, Aziz MA, Swinne D, Verhaegen J. AIDS-associated cryptococcal meningitis in Rwanda (1983-1992): epidemiologic and diagnostic features. J Infect 1999; 39: 32-37. 
[5] Gordon SB, Walsh AL, Chaponda M, Gordon MA, Soko D, Mbwvinji M, et al. Bacterial meningitis in Malawian adults: pneumococcal disease is common, severe, and seasonal. Clin Infect Dis 2000; 31: 53-57.

[6] Helbok R, Pongpakdee S, Yenjun S, Dent W, Beer R, Lackner $P$, et al. Chronic meningitis in Thailand. Clinical characteristics, laboratory data and outcome in patients with specific reference to tuberculosis and cryptococcosis. Neuroepidemiology 2006; 26: 37-44.

[7] Heyderman RS, Gangaidzo IT, Hakim JG, Mielke J, Taziwa A, Musvaire P, et al. Cryptococcal meningitis in human immunodeficiency virus-infected patients in Harare, Zimbabwe. Clin Infect Dis 1998; 26: 284-289.

[8] Hovette P, Soko TO, Raphenon G, Camara P, Burgel PR, Garraud O. Cryptococcal meningitis in AIDS patients: an emerging opportunistic infection in Senegal. Trans R Soc Trop Med Hyg 1999; 93: 368.

[9] Schutte CM, Van der Meyden CH, Magazi DS. The impact of HIV on meningitis as seen at a South African Academic Hospital (1994 to 1998). Infection 2000; 28: 3-7.

[10] Okongo M, Morgan D, Mayanja B, Ross A, Whitworth J. Causes of death in a rural, population-based human immunodeficiencyvirus type 1 (HIV-1) natural history cohort in Uganda. Int J Epidemiol 1998; 27: 698-702.

[11] French N, Gray K, Watera C, Nakiyingi J, Lugada E, Moore $\mathrm{M}$, et al. Cryptococcal infection in a cohort of HIV-1-infected Ugandan adults. AIDS 2002; 16: 1031-1038.

[12] Corbett EL, Churchyard GJ, Charalambos S, Samb B, Moloi V, Clayton TC, et al. Morbidity and mortality in South African gold miners: impact of untreated disease due to humanimmunodeficiency virus. Clin Infect Dis 2002; 34: $1251-1258$

[13] Shih CC, Chen YC, Chang SC, Luhi KT. Hsieh WC. Cryptococcal meningitis in non-HIV-infected patients. $Q J$ Med 2000; 93: 245-251.

[14] Lui G, Lee N, Ip M., Choi KW, Tso YK, Lam E, et al. Cryptococcosis in apparently immunocompetent patients Q J Med 2006; 99: 143-151.

[15] Bello YB, Machado HG, Silveira JF, Schettini F, Junior GM, Junior SD et al., Cryptococcal meningitis in immunocompetent patient-case report. American Medical Journal 2013; 4: 100-104.

[16] Lester FT. Cryptococcal meningitis complicating remoteskullfracture J. Neurology, Neurosurgery, and Psychiatry, 1978; 41: 672-673.

[17] Gebremedhin ACryptococcal meningitis in a young Ethiopian woman with AIDS. Ethiop Med J. 199; 1; 30: 169-73

[18] Teshale S, Shitaye A., Abraham A, Atakilti A, Ermias D. Cryptococcal meningitis in patients with acquired immunodeficiency syndrome in pre-HAART era at Gondar College of Medical Sciences Hospital north-west Ethiopia. Ethiop Med, J, 2010; 48: 5-15.
[19] Alemu AS, Russell R, Kempker, Admasu T, Smitson C., Nega $\mathrm{B}$ et al. High Prevalence of Cryptococcal Antigenemia among HIV-infected Patients Receiving Antiretroviral Therapy in Ethiopia. PLoS ONE 8 (3): e58377.

[20] Tafese B, Yimtubezinash W, Daniel A, Gonfa A, David Boulware R. Comparison of Cryptococcal Antigenemia between Antiretroviral Naı"ve and Antiretroviral Experienced HIV Positive Patients at Two Hospitals in Ethiopia. PLoS ONE 8 (10): e75585.

[21] Chen S, Sorrell T, Nimmo G, Speed B, Currie B, Ellis D et al. Epidemiology and host- and variety-dependent characteristics of infection due to Cryptococcus neoformans in Australia and New Zealand. Australasian Cryptococcal Study Group. Clin Infect Dis 2000; 31: 499-508.

[22] Friedman GD, Jeffrey Fessel W, Udaltsova NV, Hurley LB. Cryptococcosis: the 1981-2000 epidemic. Mycoses 2005; 48: $122-125$.

[23] Bergemann A, Karstaedt AS. The spectrum of meningitis in a population with high prevalence of HIV disease. QJM. 1996; 499-504.

[24] Hakim JG, Gangaidzo IT, Heyderman RS, Mielke J, Mushangi E, Taziwa A, et al. Impact of HIV infection on meningitis in Harare, Zimbabwe: a prospective study of 406 predominantly adult patients. AIDS. 2000; 14: 1401-7.

[25] Silber E, Sonnenberg P, Ho KC, Koornhof HJ, Eintracht S, Morris L, et al. Meningitis in a community with a high prevalence of tuberculosis and HIV infection. J Neurol Sci. $1999 ; 162: 20-6$

[26] Jarvis JN, Meintjes G, Williams A, Brown Y, Crede T, Harrison TS. Adult meningitis in a setting of high HIV and TB prevalence: findings from 4961 suspected cases. BMC Infect Dis. 2010; 10: 67.

[27] Cohen DB, Zijlstra EE, Mukaka M, Reiss M, Kamphambale $\mathrm{S}$, Scholing $\mathrm{M}$, et al. Diagnosis of cryptococcal and tuberculosis meningitis in a resource-limited African setting. Trop Med Int Health. 2010; 15: 910-917.

[28] Mwaba P, Mwansa J, Chintu C, Pobee J, Scarborough M, Portsmouth S, Zumla A,. Clinical presentation, natural history, and cumulative death rates of 230 adults with primary cryptococcal meningitis in Zambian AIDS patients treated under local conditions. Postgrad Med J 2001; 77: 769-77.

[29] Mahale K, S Patil S, Ravikumar, Nagabhushan, Ramanath Mahale R, prevalence of cryptococcal meningitis among immunocompetent and immunocompromised individuals in Bellary, South India - a Prospective Study Journal of Clinical and Diagnostic Research. 2012 May (Suppl-1), 6: 388-392.

[30] Pappas PG, Perfect JR, Cloud GA, Larsen RA, Pankey GA, Lancaster DJ et al Cryptococcosis in human immunodeficiency virus-negative patients in the era of effective azole therapy. Clin Infect Dis 2001; 33: 690-699.

[31] Prasad KN, Agarwal J, Nag VL, Verma AK, Dixit AK, Ayyagari A Cryptococcal infection in patients with clinically diagnosed meningitis in a tertiary care center. Neurology India, 2003; 51: 364-366. 